\title{
Effects of Annealing on Chemical-Vapor Deposited PureB Layers
}

\author{
K. R. C. Mok, ${ }^{a, z}$ A. H. G. Vlooswijk, ${ }^{b}$ V. Mohammadi, ${ }^{a}$ and L. K. Nanver ${ }^{a}$
}

${ }^{a}$ Department of Microelectronics, Delft University of Technology, 2628CT Delft, The Netherlands

${ }^{b}$ Tempress Systems BV, 8171MD Vaassen, The Netherlands

Chemical-vapor-deposited pure boron (PureB) layers can be used as a source of p-type boron dopants for thermal diffusion into silicon during a drive-in anneal. In this work, the effect of thermally annealing PureB layers is investigated in terms of surface morphology and electrical properties. The presence of a few nanometer-thick PureB layer on the Si surface was found to increase the silicon oxide growth rate by several factors during annealing in an oxygen-containing atmosphere. The oxide thickness was dependent on the initial PureB layer thickness and oxygen concentration during anneal. In an oxygen-limited atmosphere, the final thickness is insensitive to the anneal temperature as the reaction is diffusion-limited and, after oxide removal, a hydrophilic boron rich layer remains on the Si surface. With a high oxygen concentration, the boron is depleted by an oxidation of the boron-doped silicon resulting in a lower surface concentration and higher sheet resistance. A reaction mechanism involving the oxidation of Si-B compounds to form $\mathrm{B}_{2} \mathrm{O}_{3}$ is proposed to explain the experimental observations. With solar cell and other photodiode applications in mind, the sheet resistance and carrier lifetime measurements were performed and show that a one-step oxidation process can simultaneously drive-in the dopants and form an oxide passivation layer.

(C) 2013 The Electrochemical Society. [DOI: 10.1149/2.044309jss] All rights reserved.

Manuscript submitted June 5, 2013; revised manuscript received July 19, 2013. Published August 2, 2013.

The chemical vapor deposition (CVD) of pure boron (PureB) layers has in recent years been successfully applied for fabricating extremely shallow, less than $10-\mathrm{nm}$ deep, silicon $p^{+} n$ junction diodes. ${ }^{1}$ This has provided impressive performance of bulk-Si photodiodes for detecting low penetration-depth beams. ${ }^{2,3}$ Ideal low-leakage diode characteristics are achieved for deposition temperatures from 400 to $700^{\circ} \mathrm{C}^{4}$ In addition, the $700^{\circ} \mathrm{C}$ PureB deposition can be deposited with well-controlled uniformity and reproducibility down to a thickness of $2 \mathrm{~nm}$ in a conformal manner with high selectivity to silicon rather than silicon oxide, ${ }^{5}$ making this technology attractive for advanced device architectures.

From studies of the quantum efficiency of VUV photodiodes ${ }^{3}$ fabricated with PureB anode regions subjected to various drive-in procedures it has been found that high efficiencies are maintained despite the increase in junction depth from a few nanometers to the hundred-nanometer range. This is accorded to the defect-free nature of the doping process and the lack of roll-off at the surface of the resulting doping profiles. These features are also attractive for the manufacture of $p^{+}$emitter layers for solar cells and for the passivation of depleted interfaces in other types of photodiode structures. A batch furnace system for the CVD of PureB is currently being developed for high-throughput industrial applications. ${ }^{6}$ For application in standard n-type crystalline silicon solar cells, substantial drive-in is required to achieve the dopant profiles and sheet resistance necessary for a low series resistance through the p-type surface layers and associated contacts. More critically, a well-passivated interface is necessary for maintaining high carrier lifetimes.

In conventional boron doping for silicon solar cells, a non-selective boron silicate glass layer is first deposited as a source of dopants for drive-in. This otherwise undesirable layer is then removed and replaced with a suitable passivation layer. PureB deposition on the other hand is selective to silicon and can be driven-in without a capping layer. The desired dopant profile and sheet resistance can be controlled by optimizing the thickness of the initial PureB layer that is the source of dopants, as well as the thermal budget of the subsequent anneal. For a high optical efficiency, it is vital to control the properties of the final surface layer and the dopant profile in the silicon. It is investigated here whether thermal oxidation and dopant drive-in can be performed simultaneously to form a good quality oxide passivation layer in one furnace step. This process would open the way to process simplifications that could lower production costs. The applicability of the fabricated layers are evaluated in terms of the electrical parameters of lifetime and sheet resistance, and the surface morphology before and after anneal/oxidation is studied to achieve an understanding of the thermal reaction mechanisms involved.

\section{Experimental}

For the surface morphology analysis and sheet resistance measurements, phosphorus-doped Czochralski wafers of (100) orientation and $1-5 \Omega \cdot \mathrm{cm}$ resistivity were used in this work. The PureB layers were deposited in an ASM single-wafer Epsilon 2000 reactor. During deposition, diborane was used as a source gas and hydrogen as the carrier gas. Since PureB selectively deposits on silicon and not on silicon oxide, it is essential for the silicon surface to be native oxide free. Therefore, the native oxide is first removed from the silicon surface by HF dip-etching and Marangoni drying before deposition. $^{7}$

In this study, PureB was deposited at $700^{\circ} \mathrm{C}$ and atmospheric pressure with different deposition times of 4,9 and $20 \mathrm{~min}$. The subsequent drive-in diffusion was done at 850,950 and $1050^{\circ} \mathrm{C}$ for $30 \mathrm{~min}$ in nitrogen ambient in a Tempress furnace system. In order to investigate the effect of oxygen concentration, annealing was performed either in-situ in the Epsilon reactor in nitrogen ambient, or during dry oxidation in the furnace system with $14 \%$ oxygen concentration (1 slm $\mathrm{O}_{2}, 6 \operatorname{sim} \mathrm{N}_{2}$ ).

Ellipsometry was used to characterize the thickness of as-deposited PureB layers and silicon oxide layers after anneal. Surface morphology of the deposited, annealed and oxidized layers was analyzed with high-resolution transmission electron microscopy (HRTEM). Electron energy loss spectroscopy (EELS) was used to determine the elemental composition of the surface layers.

The samples prepared for HRTEM were partially textured with inverted pyramids. This was done by first growing a 300 -nm-thick thermal oxide layer on bare silicon wafers. Squares 10-micrometer wide were then plasma-etched to expose the silicon. The samples were dipped in a $0.55 \% \mathrm{HF}$ solution for 4 minutes to remove native oxide and the exposed silicon was subsequently chemically etched by $2 \%$ tetramethylammonium hydroxide (TMAH), $10 \%$ isopropyl alcohol (IPA) in deionized water at $80^{\circ} \mathrm{C}{ }^{8}$ The silicon oxide acts as a mask while the TMAH solution preferentially etches silicon along the (100) and (110) crystallographic planes, resulting in (111) surfaces being exposed on the textured surface.

For lifetime measurements, phosphorus-doped monocrystalline float zone (FZ), double-side polished wafers of (100) orientation, $1-5 \Omega \cdot \mathrm{cm}$ resistivity and $260-300 \mu \mathrm{m}$ thickness were used. To form symmetrical $\mathrm{p}^{+} / \mathrm{n} / \mathrm{p}^{+}$samples, PureB was deposited on one side, and then the wafer was manually flipped ex-situ and immediately processed with the same recipe on the other side. The second exposure of the first side to $700^{\circ} \mathrm{C}$ gives a very limited drive-in of boron into the $\mathrm{Si}$ as set by the solid solubility of $2 \times 10^{19} \mathrm{~cm}^{-3}$. This effect is negligible compared to the effect of the subsequent anneal/oxidation. The bulk effective carrier lifetimes were measured using the transient photo-conductance method. 

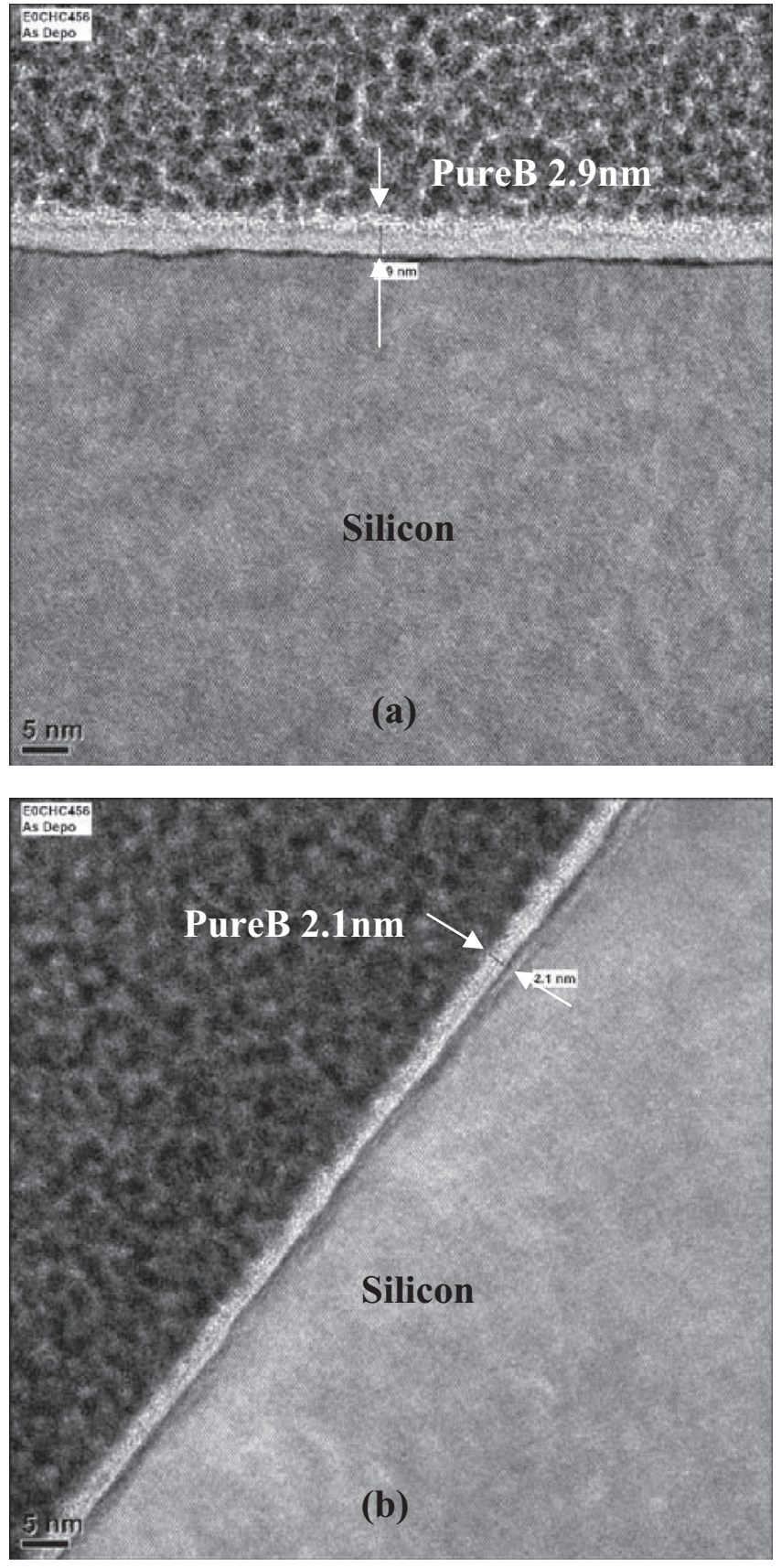

Figure 1. HRTEM image of an as-deposited PureB layer formed by a 9-min deposition time on a (100) surface (a) and on a (111) surface formed after TMAH texturing (b).

\section{Results and Discussion}

The surface morphology of the as-deposited, annealed and oxidized layer was analyzed with High-Resolution Transmission Electron Microscopy (HRTEM). Figure 1 shows an image of the PureB layer deposited with a 9-min deposition time, resulting in a $\sim 3$-nmthick PureB layer. On the (111) surfaces resulting from texturing by TMAH, the PureB layer is conformal but has a slightly lower thickness than the non-textured (100) surface.

Figure 2 shows an image following a furnace anneal at $950^{\circ} \mathrm{C}$ for $30 \mathrm{~min}$ in nitrogen ambient, whilst Figure 3 shows an image following a dry oxidation at $950^{\circ} \mathrm{C}$ for $30 \mathrm{~min}$ in $14 \%$ oxygen concentration. On the $\mathrm{Si}$, an amorphous layer can be seen to have been formed in both cases. Based on Electron Energy Loss Spectroscopy (EELS) charac-
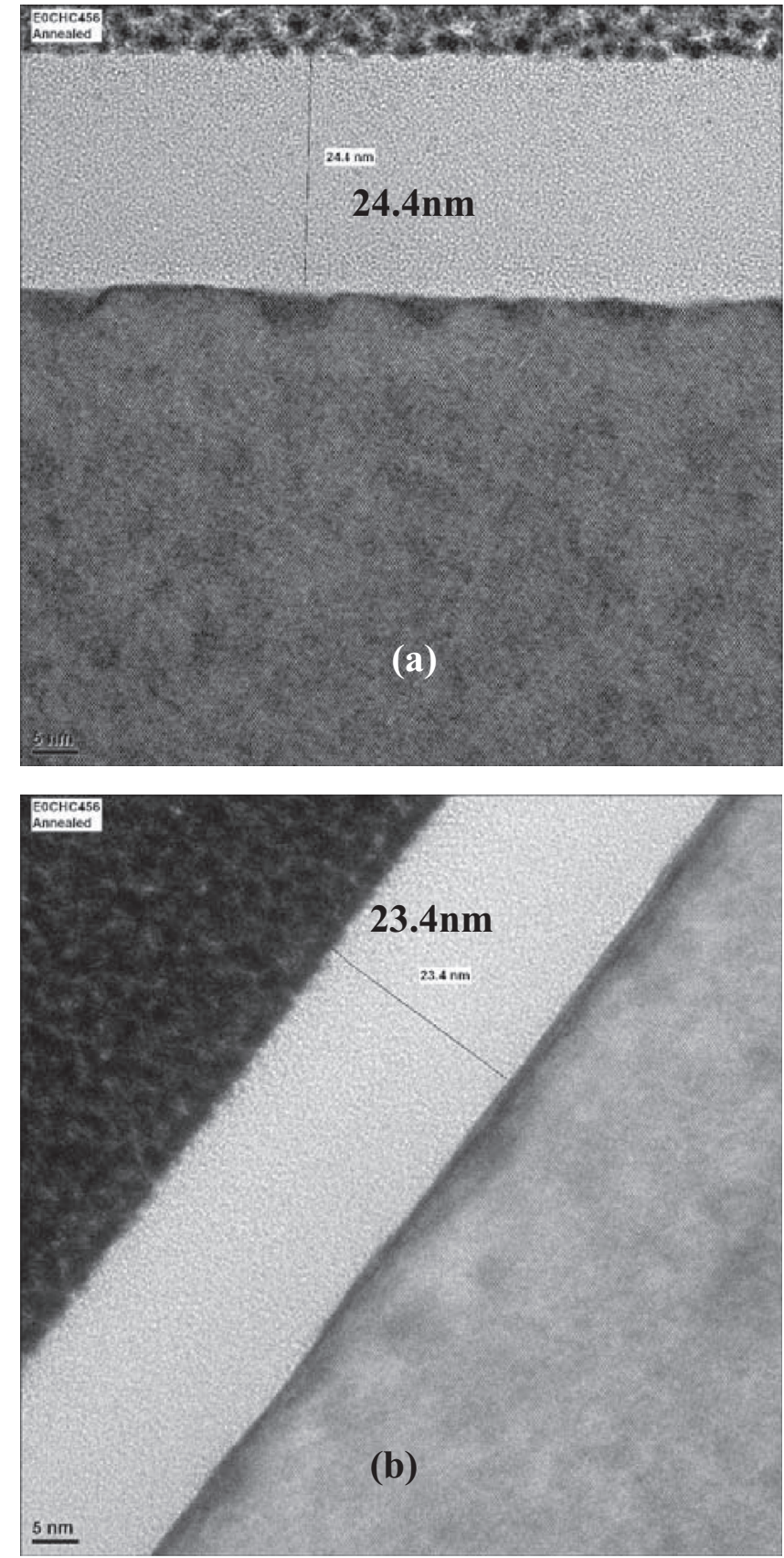

Figure 2. HRTEM image of a PureB layer formed by a 9-min deposition followed by a furnace anneal at $950^{\circ} \mathrm{C}$ for $30 \mathrm{~min}$ in nitrogen ambient on a (100) surface (a) and on a (111) surface formed after TMAH texturing (b).

terization, the elemental composition of this layer is predominantly silicon, followed by oxygen, with trace amounts of boron. Apparently, a silicon oxide layer is grown in both cases. For the annealed sample of Figure 2, this layer grows in an oxygen limited atmosphere and, in contrast to the sample of Figure 3, a clear peak in boron signal is observed at the $\mathrm{SiO}_{2}$-Si interface.

For the anneal in nitrogen ambient the furnace is known to not be completely oxygen-free but the resulting oxide thickness of $\sim 24 \mathrm{~nm}$ is impressive since it would otherwise be barely significant. During the dry oxidation with $14 \%$ oxygen concentration, the oxide layer was even thicker, $\sim 40 \mathrm{~nm}$, as compared to $\sim 9 \mathrm{~nm}$ on a control sample of bare (100) silicon. Moreover, it is generally understood that, as compared to (100) surfaces, the (111) silicon surface has a higher oxide growth rate due to the higher silicon atomic 

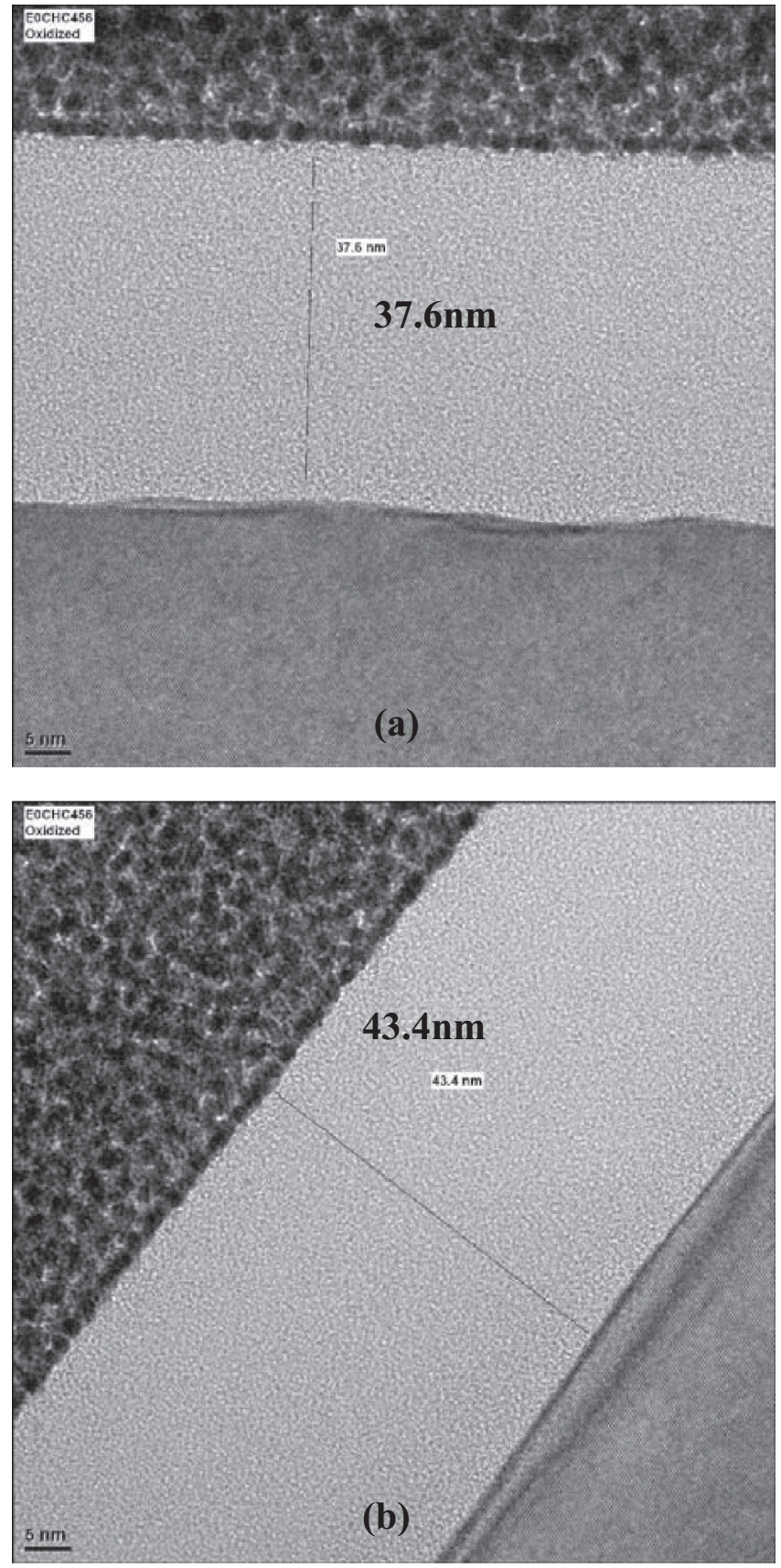

Figure 3. HRTEM image of a PureB layer formed by a 9-min deposition followed by dry oxidation at $950^{\circ} \mathrm{C}$ for $30 \mathrm{~min}$ in $14 \%$ oxygen concentration on a (100) surface (a) and on a (111) surface formed after TMAH texturing (b).

density. ${ }^{9}$ However, from the HRTEM images, it is clear that the oxide surface layers are conformal on both the (100) and (111) surfaces and very nearly the same thickness. All in all, these results suggest that the presence of boron is very strongly influencing the rate of oxidation.

In Fig. 4 the relationship is plotted between the oxide thickness after various 30-min thermal anneal temperatures in nitrogen ambient and the original PureB layer thickness. All the layer thicknesses were measured by ellipsometry and it should be noted that these measurements are well-corroborated by the HRTEM images. The PureB layer thickness increases linearly with deposition time with a deposition rate of about $0.4 \mathrm{~nm} / \mathrm{min}$. Wafers from two different runs are included in the plot. Those from the first run (run 1) received anneal

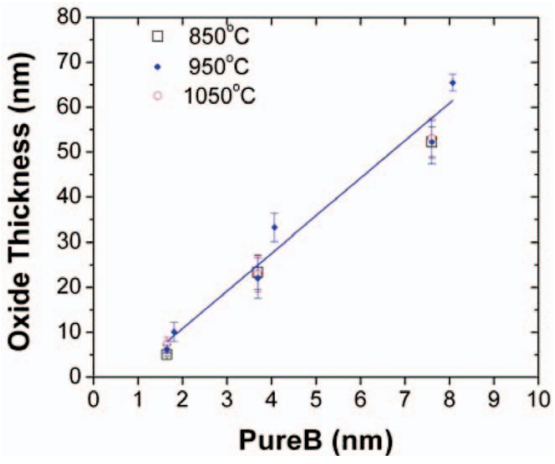

Figure 4. Oxide thickness resulting from a 30-min anneal in nitrogen ambient at different temperatures as a function of the original PureB thickness.

temperatures of 850,950 and $1050^{\circ} \mathrm{C}$ while those from the second run (run 2) were only annealed at $950^{\circ} \mathrm{C}$. The data points from this second run are shifted slightly higher with respect to the first one, both with respect to PureB thickness and final oxide thickness. For the first run, where the three anneal temperatures are performed on wafers that are known to have exactly the same PureB thicknes, the resulting oxide thickness is also practically identical. Therefore, it is clear from Fig. 4 that, not only is the oxide thickness strongly dependent on the initial PureB layer thickness, but it also appears to be linearly related to this thickness with values that are independent of the annealing temperature. In contrast, for a bare Si wafer the anneal in nitrogen only results in an oxide coverage comparable to a native oxide and this is too thin to be measured by our ellipsometry system.

In order to investigate the effect of oxygen concentration during anneal, an in-situ anneal was performed in the Epsilon reactor in nitrogen ambient. The carrier gases used in the Epsilon reactor pass through a purifier and have impurity levels below $10 \mathrm{ppb}$. Therefore, PureB deposition and in-situ thermal annealing takes place under practically oxygen-free conditions and in this case, no oxide layer could be detected by ellipsometry.

More details of the surface layers were obtained by testing with chemical etchants. It is known that silicon containing boron layers are resistant to various acidic solutions like $\mathrm{H}_{2} \mathrm{SO}_{4}$, $\mathrm{HF}$ and $\mathrm{H}_{3} \mathrm{PO}_{4}$ due to the low solubility of the layers. ${ }^{10}$ However, it should be noted that the PureB layer can be removed by hot $\mathrm{HNO}_{3}$ and the boron-rich silicon layer is then oxidized by $\mathrm{HNO}_{3}$. Therefore, repeated $\mathrm{HNO}_{3}$ and HF treatments allow for a removal of the boron-rich layer. ${ }^{11}$ As a chemical etch test, the various samples were subjected to a 10min $0.55 \% \mathrm{HF}$ etch treatment. The Epsilon in-situ annealed sample remained hydrophilic due to the presence a boron-rich layer. The sample annealed in nitrogen ambient remained hydrophilic in large patches as well, although ellipsometry measurement showed that the oxide layer was effectively etched away. The hydrophilic nature of the silicon surface implies the existence of a boron-rich layer, as is consistent with EELS results which showed a boron peak at the $\mathrm{Si}-\mathrm{SiO}_{2}$ interface. Finally, the sample that underwent dry oxidation became hydrophobic after a $10-\min 0.55 \% \mathrm{HF}$ etch, which indicates that the etched surface was free of both oxide and boron-rich layers.

In the in-situ anneal case, boron atoms can react with silicon atoms at the surface of the silicon upon high-temperature annealing. Based on work done on molecular layer doping, Saitoh et al. analyzed the composition of a pure boron layer after annealing at $900^{\circ} \mathrm{C}$ using Rutherford backscattering (RBS) analysis and they estimated the $\mathrm{Si}-\mathrm{B}$ compound to be $\mathrm{SiB}_{6} .{ }^{11}$ The composition of $\mathrm{Si}-\mathrm{B}$ compound has been widely investigated and different compositions have been suggested. ${ }^{11-13}$ As a result, it is generally referred to as the boron-rich layer. This boron-rich layer acts as a source of dopants from which boron atoms thermally diffuse into the silicon bulk as governed by the concentration gradient and the solid solubility.

The same reactions of B with the Si surface occur in the furnace system. However, in the presence of oxygen, the following additional 
reactions take place: ${ }^{12-13}$

$$
\begin{gathered}
4 \mathrm{~B}+3 \mathrm{O}_{2} \rightarrow 2 \mathrm{~B}_{2} \mathrm{O}_{3} \\
\mathrm{Si}+\mathrm{O}_{2} \rightarrow \mathrm{SiO}_{2} \\
2 \mathrm{SiB}_{6}+11 \mathrm{O}_{2} \rightarrow 6 \mathrm{~B}_{2} \mathrm{O}_{3}+2 \mathrm{SiO}_{2} \\
2 \mathrm{~B}_{2} \mathrm{O}_{3}+3 \mathrm{Si} \rightarrow 4 \mathrm{~B}+3 \mathrm{SiO}_{2}
\end{gathered}
$$

Initially, $\mathrm{B}$ or $\mathrm{SiB}_{6}$ in the boron-rich layer reacts with oxygen to form $\mathrm{B}_{2} \mathrm{O}_{3}$. Silicon from the bulk then diffuses to the surface to react with $\mathrm{B}_{2} \mathrm{O}_{3}$ to form silicon oxide and the resulting boron can diffuse into the bulk silicon. This reaction mechanism explains why the initial PureB layer thickness determines the final silicon oxide thickness as seen in Fig. 4. In addition, during furnace annealing in a nitrogen ambient whereby the supply of oxygen to the silicon surface is limited, $\mathrm{Si}-\mathrm{B}$ compounds or a boron-rich layer may remain at the $\mathrm{Si}$ $\mathrm{SiO}_{2}$ interface. This appears to happen in our case since such a residual B-layer is consistent with EELS results and the chemical etch tests. Furthermore, as the source of oxygen is very limited, the reaction becomes diffusion-limited, which explains the negligible effect of temperature on the final oxide thickness.

At sufficiently high oxygen concentration, the $\mathrm{Si}-\mathrm{B}$ compound is completely oxidized preventing the formation of a hydrophilic boronrich layer. ${ }^{12-14}$ Moreover, the reaction can be expected to become reaction-limited, whereby the annealing temperature becomes a factor in the final oxide thickness. This type of behavior can be deduced from Figure 5 where a plot is shown of the oxide thickness resulting from 20-min PureB deposition followed by 30-min anneal in nitrogen ambient or 10-min dry oxidation in $14 \%$ oxygen as a function of furnace temperatures. On the one hand, the oxide thickness resulting from the 30-min furnace anneal in nitrogen ambient is constant even up to $1050^{\circ} \mathrm{C}$, and on the other, in the presence of $14 \%$ oxygen concentration, the oxide thickness increases with temperature. It should be noted the oxide thickness resulting from annealing in nitrogen ambient is not influenced by the loading and unloading temperature of the wafers. Both $800^{\circ} \mathrm{C}$ and a lower temperature of $600^{\circ} \mathrm{C}$ show the same trends, implying that the loading and unloading conditions are unimportant for achieving an oxide thickness that is unrelated to the annealing temperature.

The diffusion of silicon from the bulk substrate into the boron-rich layer has previously been observed to result in large spike formation at the edges and corners of $\mathrm{SiO}_{2}$-isolation windows to the $\mathrm{Si}$ substrate when a PureB layer was deposited and exposed to thermal drive-in at $1100^{\circ} \mathrm{C}$ for $195 \mathrm{~min} .{ }^{15}$ It has been proposed that the large spikes were formed because, at such elevated temperatures, the $\mathrm{Si}$ reacts with $\mathrm{SiO}_{2}$ at the window edges to form volatile $\mathrm{SiO} .{ }^{16}$ This results in both

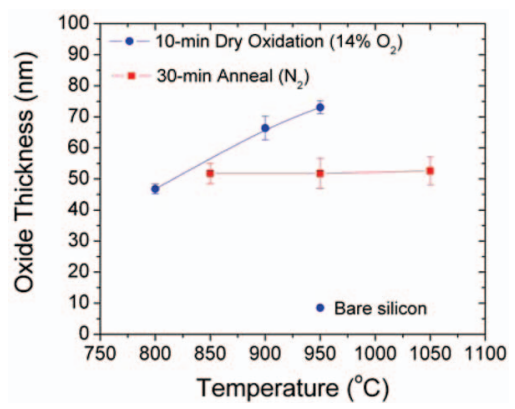

Figure 5. Oxide thickness as a function of the anneal temperature resulting from a 20-min PureB deposition followed either by a 30-min furnace anneal

\begin{tabular}{|c|c|c|c|}
\hline Sample & Process Condition & $\begin{array}{c}\text { Sheet Resistance } \\
{[\Omega / \mathrm{sq}]}\end{array}$ & Lifetime $[\mu s]$ \\
\hline 1 & $\begin{array}{l}\text { PureB } 9 \text { min Deposition } \\
\text { Anneal }\left(\mathrm{N}_{2}\right) 950^{\circ} \mathrm{C} 30 \mathrm{~min} \\
\text { Chemical Etch } \mathrm{HNO}_{3}+\mathrm{HF} \\
\text { Oxidation } 800^{\circ} \mathrm{C} 10 \mathrm{~min}\end{array}$ & 61.2 & 257 \\
\hline 2 & $\begin{array}{l}\text { PureB } 9 \text { min Deposition } \\
\text { Oxidation } 950^{\circ} \mathrm{C} 30 \mathrm{~min}\end{array}$ & 205 & 251 \\
\hline 3 & $\begin{array}{l}\text { PureB } 9 \text { min Deposition } \\
\text { Oxidation } 950^{\circ} \mathrm{C} 10 \mathrm{~min}\end{array}$ & 136 & 353 \\
\hline
\end{tabular}
in nitrogen ambient or a 10 -min dry oxidation in $14 \%$ oxygen. The oxide thickness formed by the latter thermal process on a bare $\mathrm{Si}$ wafer is also included. spiking and a lateral widening of the windows. These detrimental effects become more severe in the presence of boron. This can be understood from the reaction mechanism, whereby the boron-rich layer in the windows reacts with small amounts of oxygen in the furnace during the drive-in to form $\mathrm{B}_{2} \mathrm{O}_{3}$. Subsequently, silicon from the window perimeter diffuses to react with $\mathrm{B}_{2} \mathrm{O}_{3}$, leaving behind large cavities or spike formation at the window edges and particularly in the corners.

An important criterion for high-efficiency Si solar cells is a high effective carrier lifetime. It has previously been reported that boronrich interface layers of more than 10-nm thick should be avoided to prevent degradation of carrier lifetime in the bulk of the silicon wafer. ${ }^{14}$ In order to test the suitability of this process for solar cell applications a few symmetrical samples were prepared as shown in Table I to evaluate the passivation quality of the surface layer. All three samples were exposed to a maximum temperature of $950^{\circ} \mathrm{C}$. For the sample 1 that first was annealed at this temperature, it was important to avoid any detrimental impact of the boron-rich layer on the effective carrier lifetimes. Therefore, the sample underwent chemical etch treatment involving boiling $\mathrm{HNO}_{3}$, followed by $\mathrm{HF}$ to remove both the oxide and boron-rich layers. Successful removal was indicated by the eventual hydrophobicity of the surface. Subsequently, a $800^{\circ} \mathrm{C} 10$-min dry-oxidation process was performed for passivation. The low thermal budget of this oxidation step compared to the diffusion step was not expected to produce any further boron diffusion of significance. As a final step, a forming gas anneal was also performed on all the samples. The lifetime values shown in Table I were measured immediately after processing and forming gas anneal to avoid inconsistent comparisons due to degradation with time, as recombination in oxide-passivated boron-diffused samples has in the past been found to increase severely with time. ${ }^{17}$ The lifetimes were obtained by the transient photoconductance method and the sheet resistances were measured on separate test wafers by 4-point probing.

From Table I, it can be seen that the lowest sheet resistance is achieved for sample 1 that was annealed in nitrogen ambient for $30 \mathrm{~min}$. Despite exposure of sample 2 to a similar thermal budget during an oxidizing step in $14 \%$ oxygen, the sheet resistance of this sample is much higher. As can be seen from Figures 2 and 3, the oxide layer resulting from this comparable treatment was $24 \mathrm{~nm}$ for sample 1 and $\sim 40 \mathrm{~nm}$ for sample 2 . This indicates that boron depletion has taken place in sample 2 as boron-doped silicon was oxidized leading to a lower surface concentration of boron and higher sheet resistance. By reducing the oxidation time to $10 \mathrm{~min}$, sample 3 has a lower sheet resistance due to a higher surface concentration. More importantly, the maximum bulk effective lifetime was obtained with sample 3, showing that the surface layer resulting from a one-step B-driven anneal/oxidation has passivating properties at least comparable to an oxide layer grown separately on a clean Si-surface as in sample 1.

In addition to optimizing the initial PureB layer thickness and thermal budget needed to reach the required boron activation, sheet resistance and junction depth, it can be concluded that the oxygen concentration during the drive-in anneal is also critical. Sufficient oxygen 
can eradicate any potential residual boron-rich layers that may otherwise degrade the bulk lifetime while excessive oxygen results in boron depletion due to oxidation of the boron-doped silicon. Therefore, a process condition in terms of oxygen concentration has to be optimized to find an optimal balance. In this respect, it should be noted that the optical properties of the surface layer will be influenced by the oxide thickness, an effect that is not considered here. However, it may also be an important factor for optimization of the solar cell efficiency.

\section{Conclusion}

In this work, it has been demonstrated that for the annealing of chemical-vapor-deposited PureB layers on $\mathrm{Si}$, the oxygen concentration during the anneal plays a critical role in determining the surface morphology and electrical properties. It was found that nanometerthick PureB layers, upon annealing in the presence of oxygen function as a catalyst for silicon oxide growth. The resulting silicon oxide thickness was shown to be strongly dependent on the original PureB layer thickness as well as the oxygen concentration during the thermal anneal. In an oxygen-limited atmosphere, the silicon oxide thickness becomes insensitive to the process temperature since the reaction is diffusion-limited. In this case, a boron-rich layer remains at the $\mathrm{SiO}_{2}$ $\mathrm{Si}$ interface after annealing. This layer proved to be hydrophilic when exposed to air by selective wet etching. On the other hand, an ample supply of oxygen during annealing results in boron depletion of the boron-doped Si surface due to an enhanced oxidization and this results in a lower surface concentration and higher sheet resistance. In terms of solar cell and other photodiode applications, the sheet resistance and lifetime results show that a one-step process can be used to simultaneously drive-in the dopants and form a good oxide passivation layer.

\section{Acknowledgments}

The authors thank the staff of the DIMES-ICP cleanroom for their support, especially Alex van den Bogaard and Cassan Visser for help with the furnace recipes and with ellipsometry. This work was supported by the Tempress Systems BLISC project.

\section{References}

1. F. Sarubbi, L. K. Nanver, and T. L. M. Scholtes, IEEE Trans. Electron Devices, 57(6), 1269 (2010)

2. A. Šakić, L. K. Nanver, G. van Veen, K. Kooijman, P. Vogelsang, T. L. M. Scholtes, W. de Boer, W. H. A. Wien, S. Milosavljević, C. Th. H. Heerkens, T. Knežević, and I. Spee, Proc. of International Electron Devices Meeting (IEDM2010), San Francisco, CA, USA, IEDM2010-712, p. 31.4.1 (2010).

3. L. Shi, S. Nihtianov, L. Haspeslagh, F. Scholze, A. Gottwald, and L. K. Naver, IEEE Trans. Electron Devices, 59(11), 2888 (2012).

4. L. K. Nanver, A. Sammak, V. Mohammadi, K. R. C. Mok, L. Qi, A. Sakic N. Golshani, J. Derakhshandeh, T. M. L. Scholtes, and W. D. De Boer, ECS Trans. 49(1), 25 (2012).

5. V. Mohammadi, W. B. de Boer, T. L. M. Scholtes, and L. K. Nanver, ECS Journal of Solid State Science and Technology, 1(1) Q16 (2012).

6. K. R. C. Mok, L. K. Nanver, W. B. de Boer, and A. H. G. Vlooswijk, in Proc. ICT.OPEN: Micro technology and micro devices (SAFE), p. 1, Veldhoven, The Netherlands (2011).

7. V. Mohannadi, W. B. de Boer, and L. K. Nanver, J. Appl. Phys. 112, 113501 (2012).

8. P. Papet, O. Nichiporuk, A. Kaminski, Y. Rozier, J. Kraiem, J.-F. Lelievre, A. Chaumartin, A. Fave, and M. Lemiti, Solar Energy Materials \& Solar Cells 90, 2319 (2006)

9. E. A. Irene, H. Z. Massoud, and E. Tierney, J. Electrochem. Soc., 133(6) 12536 (1986).

10. K. M. Busen, W. A. Fitzgibbons, and T. Kloffenstein, Electrochem. Technol., 6, 256 (1968).

11. N. Saitoh, T. Akamine, K. Aoki, and Y. Kojima. Jpn. J. Appl. Phys., 32(4), 4404 (1993).

12. E. Arai, H. Nakamura, and Y. Terunuma, J. Electrochem. Soc.: Solid State Sci. Technol., 120, 980 (1973).

13. M. A. Kessler, T. Ohrdes, B. Wolpensinger, and N.-P. Harder, Semicond. Sci. Technol. 25, 055001 (2010).

14. J. Libal, R. Petres, R. Kopecek, G. Hahn, K. Wambach, and P. Fath, in Conf. Rec. of the $31^{\text {st }}$ IEEE Photovoltaic Specialists Conference, 1209 (2005)

15. P. Maleki, T. L. M. Scholtes, M. Popadić, F. Sarubbi, G. Lorito, S. Milosavljevic, W. B. de Boer, and L. K. Nanver, in $10^{\text {th }}$ International Workshop on Junction Technology (IWJT), 1, Shanghai, China (2010).

16. R. Tromp, G. W. Rubloff, P. Balk, F. K. LeGoues, and E. J. van Loenen, Phys. Rev. Lett. 55, 2332 (1985).

17. A. F. Thomson and K. R. McIntosh, Appl. Phys. Lett. 95, 052101 (2009). 